\title{
ROAD CLASSIFICATION AND CONDITION DETERMINATION USING HYPERSPECTRAL IMAGERY
}

\author{
M. Mohammadi \\ Department of Geomatics, Computer Science and Mathematics, University of Applied Sciences Stuttgart \\ Schellingstraße 24, D-70174 Stuttgart, Germany - mohammadi_2025@yahoo.com
}

KEY WORDS: Hyperspectral, Urban, ALK vector data, Classification, Condition

\begin{abstract}
:
Hyperspectral data has remarkable capabilities for automatic identification and mapping of urban surface materials because of its high spectral resolution. It includes a wealth of information which facilitates an understanding of the ground material properties. For identification of road surface materials, information about their relation to hyperspectral sensor measurements is needed. In this study an approach for classification of road surface materials using hyperspectral data is developed. The condition of the road surface materials, in particular asphalt is also investigated. Hyperspectral data with $4 \mathrm{~m}$ spatial resolution of the city of Ludwigsburg, Germany consisting of 125 bands (wavelength range of $0.4542 \mu \mathrm{m}$ to $2.4846 \mu \mathrm{m}$ ) is used. Different supervised classification methods such as spectral angle mapper are applied based on a spectral library established from field measurements and in-situ inspection. It is observed that using the spectral angle mapper approach with regions of interest is helpful for road surface material identification. Additionally, spectral features are tested using their spectral functions in order to achieve better classification results. Spectral functions such as mean and standard deviation are suitable for discriminating asphalt, concrete and gravel. Different asphalt conditions (good, intermediate and bad) are distinguished using the spectral functions such as mean and image ratio. The mean function gives reliable results. Automatisierte Liegenschaftskarte (ALK) vector data for roads is integrated in order to confine the analysis to roads. Reliable reference spectra are useful in evaluation of classification results for spectrally similar road surface materials. The classification results are assessed using orthophotos and field visits information.
\end{abstract}

\section{INTRODUCTION AND RELATED RESEARCH}

Comprehensive information about road networks as one of the transportation features is helpful for assessment and planning of transportation (routing). Retrieval of road information such as road surface material and pavement type condition is one of the essential issues in urban areas. This is done with either traditional surveying or remote sensing (RS) (Zhang and Couloigner, 2004).The former needs more labour and is more time consuming in comparison to the latter. Hyperspectral imagery, also known as imaging spectrometry, is the acquisition of data in many narrow, contiguous spectral bands (Goetz et al., 1985). It provides more detailed information in comparison to other remote sensing techniques. Different chemical materials such as asphalt and gravel by their corresponding physical (absorption, albedo, reflectivity etc) properties can be derived on a very detailed level from the hyperspectral imagery. This characteristic is helpful in discrimination and extraction of urban area objects especially those with similar spectral properties. Road surface materials can be identified with hyperspectral imagery with less cost compared to field surveying. Most of the available methods for mapping roads are either manual or semi-automatic. However, these approaches are time consuming and expensive. In particular, that they may involve a lot of field work and interpretation of aerial imagery from which only limited information can be acquired. Hyperspectral data has significant potential in terms of automatic identification of road surface materials. However, no standard approach for mapping road surfaces and identifying the condition of road surface materials exists up to date. Most of the methods that exist were originally developed for mineral detection. Thus it is a challenge to use these methods in identifying road surface materials due to the variation of these materials in relatively small regions in the case of roads.

In recent times there has been an increase in the demand to find economical automated methods to extract information from hyperspectral data due to the fast developments in urban areas. Noronha et al., (2002) focus on extraction of road centerlines, detecting pavement condition and developing a spectral library. The analysis of hyperspectral data is done using MultiSpec software and maximum likelihood classification is applied. Bhattacharyya distance is used for separability analysis between road materials and roof types. For better discrimination of roofs and roads, an object-oriented image classification technique is used. This technique tries to analyse the homogenous image object rather than independent pixels. A multispectral sensor (IKONOS) is used as an ancillary sensor. Comparison of configuration for urban target separation in spectrometry and multispectral remote sensing shows that some of the features are not determined in the latter. This is due to the broadness and location of the bands. Moreover, its broad band channels do not resolve small-scale spectral absorption features which are unique for several built up areas. According to the separability analysis results, concrete and 
gravel roads are easily distinguishable from asphalt roads. It is not easy to differentiate between some roads and roof types such as dark new roads and dark tile roofs. However, there are specific absorption features for each urban object in the short wave infrared which help in differentiating them. Segl et al. (2003) confirm that it is a challenge to use these methods in identifying urban surface materials due to the variation of these materials in relatively small regions. A hyperspectral pixel in an urban scene is generally a mixture of different material components which makes it difficult to discriminate successfully between certain classes of materials (Bhaskaran and Datt 2000).

Most of recent methods for analysis of hyperspectral data do not directly determine the materials and just define how similar the unknown material is to the known material. There are other techniques which are able to directly identify the material using spectral features. A lot of research has been done for applying these techniques to image spectrometry to have better classification results. Freek van der Meer (2004) develops a method for the analysis of hyperspectral images using absorption band depth and position over mainly mineral material. This method consists of a simple linear regression formula to estimate absorption-band parameters from hyperspectral image data which is easily implemented in ENVI tools for those users that are not familiar with programming languages. The sensitivity analysis shows that more reliable results would be achieved by more accurate absorption band parameters (shoulders, absorption points). Heiden et al. (2007) develop an approach for urban feature identification using specific robust characteristics. These spectral characteristics include absorption bands (depth and position) or sharp increase and decrease of reflectance. Separability analysis is used to evaluate the robustness of spectral features. It is concluded that urban materials need to be described by more than one type of feature. A similar approach is followed in this research. It could be helpful in identifying road surface materials since the focus is on special features such as absorption depth which may be unique for a particular material. For a comprehensive review of related work please refer to Mohammadi (2011). So far there is no standard approach to produce reasonable results. The analysis techniques for hyperspectral data can be unsupervised or supervised. Supervised techniques include similarity measures such as spectral angle mapper (SAM), spectral information divergence (SID) and spectral correlation mapper (SCM). Spectral similarity measures are used to identify unknown spectra based on a comparison with reference spectra. In addition, unique spectral characteristics within certain wavelength ranges of some spectra can be used directly to identify materials.

In the following chapters the data of the study area are described. For the classification of roads using hyperspectral data various methods have been investigated but only those which led to most successful experimental results are discussed. The analysis on the road surface condition is focused on asphalt roads with good success in identifying roads with good, intermediate and bad surface condition.

\section{STUDY AREA; HYPERSPECTRAL IMAGE DATA AND PREPROCESSING}

The analysis of hyperspectral data to support road surface material classification is done for a case study in the city of Ludwigsburg. This city is situated in Baden-Württemberg, Germany, in particular in the north part of Stuttgart region, near to river Neckar. The imagery data covers the urban area extending approximately $11 \mathrm{~km} \times 16 \mathrm{~km}$ of this city. The hyperspectral data was acquired during the HyMap campaign on 20th August, 2010 by German Aerospace Center (DLR) and consists of six strips. The data consists of 125 bands (ranging from $0.4 \mu \mathrm{m}$ to $2.5 \mu \mathrm{m}$ ) and has a ground sample distance of $4 \mathrm{~m}$. ALK vector data for roads is provided by Fachbereich Stadtplanung und Vermessung der Stadt Ludwigsburg. The vector data is used for limiting the analysis to roads. Information on road condition based on field visits is available from the municipality of Ludwigsburg and the Vermessungsbüro Praxl und Partner GmbH. The preprocessing of the hyperspectral data has been done by DLR. The data is corrected for radiometric, geometric and atmospheric effects. A high resolution LiDAR surface model of Ludwigsburg with $2 \mathrm{~m}$ raster size was made available for this purpose.

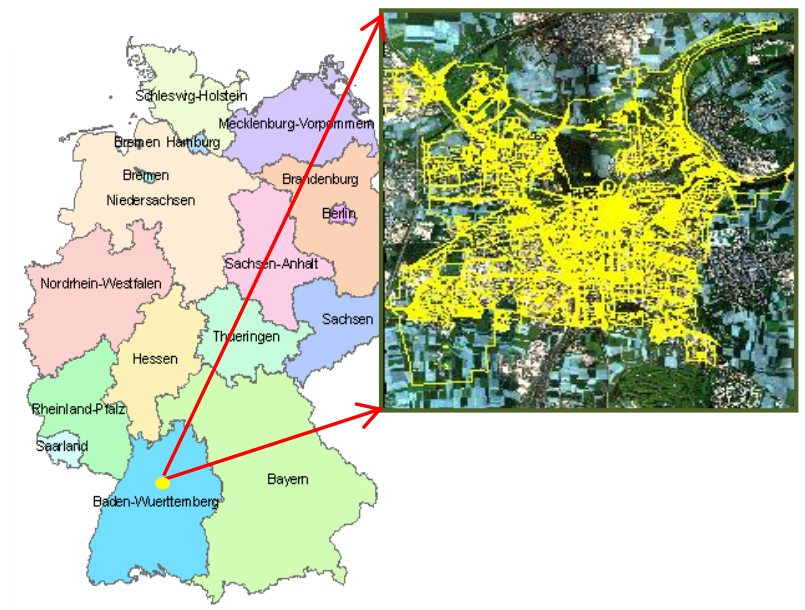

Figure 1 . Research area, Ludwigsburg.

In order to determine how well the HyMap and vector data fit, the two data sets are overlaid. It is observed that there is a shift between the two data sets in the order of $10 \mathrm{~m}$. The vector layer for roads is selected as a source of ground control points. The GCPs are used to georeference or correct the geographic location of the HyMap data. After performing an affine transformation, it is observed that an overall RMS error of about 0.7 pixels is obtained. A road mask is created from the road vector data. However, it is observed that the road mask also covers some areas with vegetation and thus these areas have to be eliminated. Vegetation spectra for different areas in the HyMap data are collected and used to create a spectral library. The HyMap data is classified using the spectral library in order to identify vegetation areas. A mask is created from the output classification map. This mask is subtracted from the road mask in order to obtain a mask limiting subsequent analyses to road surfaces only. 


\section{METHODS AND RESULTS}

In order to identify road material types, different supervised classification approaches are tested. The investigation is in two parts. The first part discusses the methods and results obtained for roads surface material identification while the second part focuses on conditions determination.

\subsection{Road surface material identification}

Asphalt or more specifically bituminous asphalt is the most used surface material of road surfaces (pavements) in Ludwigsburg. It is a composite material of construction aggregate (e.g. gravel, crushed stone, stand, etc.) and asphalt which serves as a binder. The mixing is formed in various ways which leads to a certain "asphalt variety" within classification. Other surfaces in the city are concrete surfaces, in particular the concrete pavers, and gravel surfaces. These three main classes, simply called 'asphalt', 'concrete' and 'gravel' are taken into account for road material identification. In order to map these materials, the SAM criterion and the brightness spectral feature are used. Training regions are selected over the calibration sites for the three materials. Each training region defines a spectrum as an ROI average. Using the ROI spectra, the roads within the investigation area are classified based on SAM with the default threshold setting (ENVI) of 0.1 radian. Angles between two spectra larger than this threshold lead to unclassified pixels. In addition to 0.1 two more spectral angle thresholds are tested: 0.08 and 0.15 (radians). Best classification results have been achieved with the lowest threshold of 0.08 but at the cost of a high percentage of unclassified pixels. The output classification map based on the threshold of 0.08 is shown in figure 2. Four different subclasses are defined for asphalt which are combined to form one asphalt class.

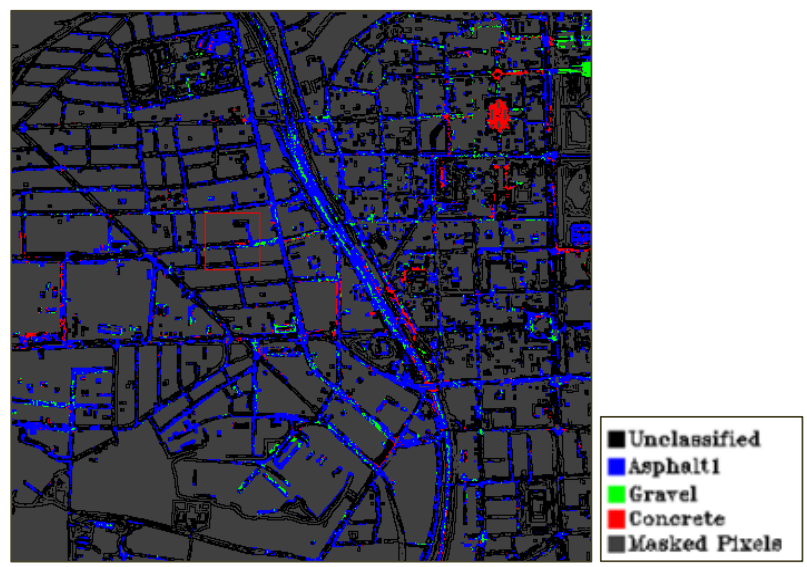

Figure 2. SAM classification using threshold of 0.08 (radians).

In the classification results (excluding non-road pixels), about $29 \%$ of the pixels are identified as asphalt, $2 \%$ as concrete, $3 \%$ as gravel and $66 \%$ of the pixels are unclassified. Figure 3 is an example of an area that shows a road segment of concrete pavers which is correctly classified. The corresponding spectra (Figure 3) indicate that the SAM similarity measure is relatively insensitive to illumination and albedo effects. Besides, field visits confirm that the material is concrete.
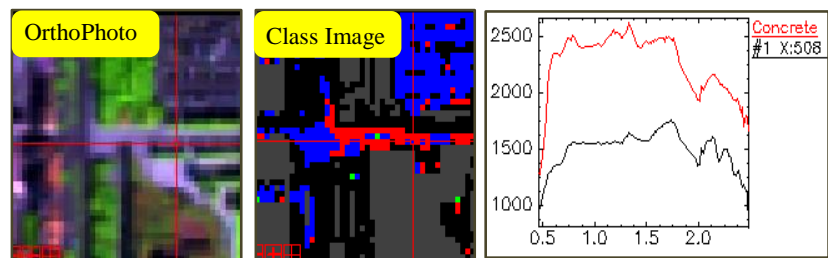

Figure 3. An example of a correctly classified area of concrete pavers.

materials involves the use of the brightness spectral feature. Brightness is one of the spectral features which is more distinct for materials with relatively flat low reflectance curves (Figure 4) such as asphalt (Heiden et al., 2005).

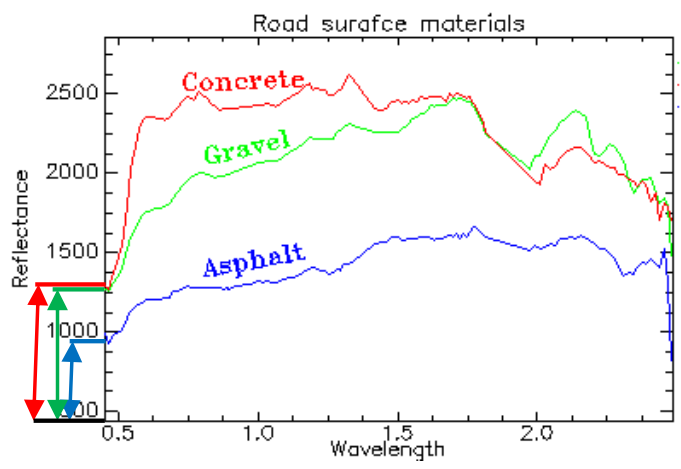

Figure 4. Asphalt, concrete and gravel spectra.

For evaluating the brightness of surface materials the mean and standard deviation functions are used. In order to distinguish asphalt, concrete and gravel, the mean function is used over the complete wavelength range of the HyMap sensor (445nm$2448 \mathrm{~nm}$ ). It is observed that asphalt has the lowest mean in this range and can be easily distinguished from the other two materials. Concrete and gravel have similar mean values over the specified wavelength range which makes it difficult to differentiate the two materials. By looking at the signatures of concrete and gravel it can be expected that the standard deviations distinguish significantly if the standard deviation function is applied over the wavelength range of $619.9 \mathrm{~nm}-1323.7 \mathrm{~nm}$. This is experimentally confirmed; in particular, concrete has a low standard deviation in this wavelength range. Based on these findings a simple procedure for the identification of asphalt, concrete and gravel is shown in figure 5 . The sequential process of applying mean and standard deviation features has a desired side effect which is the detection of vegetation pixels (high standard deviation) which might have remained after the first attempt of vegetation removal. 


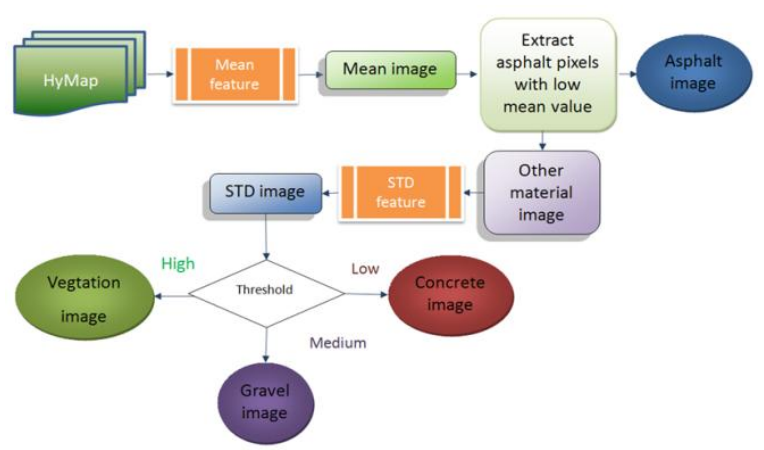

Figure 5. Classification using the brightness (mean and standard deviation) feature.

The classification result based on this procedure is shown in figure 6 for a large area. A closer look to a detail (Figure 7) reveals the high quality of the classification.

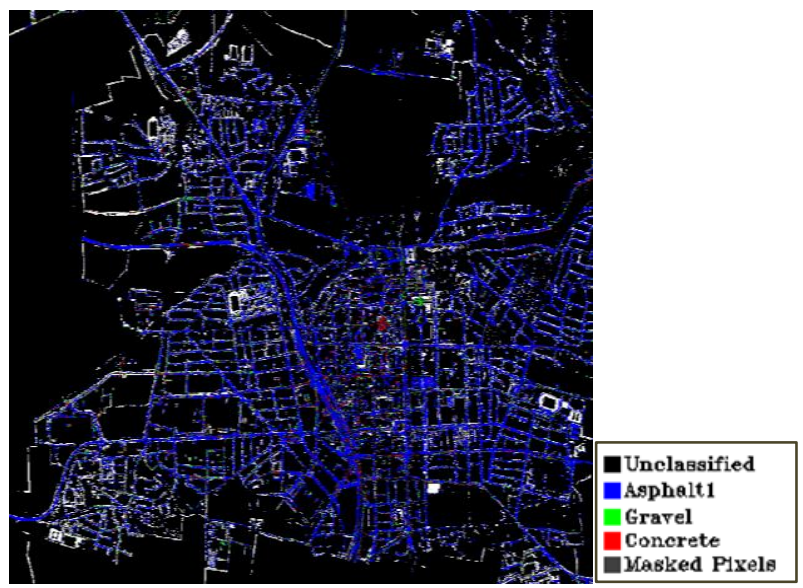

Figure 6. Classification map (mean and standard deviation functions).

In the classification results (excluding non-road pixels), $42.0 \%$ of the pixels are identified as asphalt, $2.3 \%$ as concrete, $3.2 \%$ as gravel and $52.5 \%$ of the pixels are unclassified. The number of unclassified pixels in the a priori given road layer is still high. It should be noted that no post processing was applied to improve this numbers. Compared to the SAM result the significant reduction of unclassified pixels is obvious.
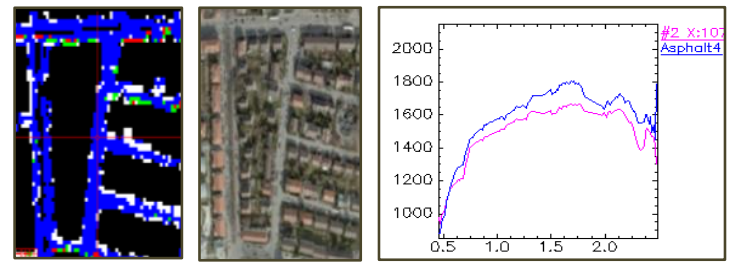

Figure 7. An example of a well classified area.

\subsection{Road surface condition determination}

In addition to the road surface material identification, condition of the road surfaces is another critical issue in relation to transportation. Recent studies (Gomez, 2002; Herold et al., 2005; Noronha et al., 2002) in hyperspectral imagery and spectrometry have shown that it is possible to map road surface condition and distress using these technologies. In terms of condition determination, the investigation is carried out for asphalt. Three categories are defined, namely good, intermediate and bad. In order to determine the different conditions according to the three defined categories, spectral features are used. The spectral features tested are brightness using mean function, decrease or increase in reflectance using the ratio function and hydrocarbon absorption bands.

Mean is one of the functions which is helpful for asphalt condition identification. New asphalt has lower mean and as the condition gets worse the mean gets higher. In terms of the ratio function (Herold et al., 2005) there are two image ratios which are significant in asphalt condition differences. These are situated in visible and short wavelength infrared bands. These ranges (Figure 8) are 490nm - 830nm for visible (VIS2) and $2120 \mathrm{~nm}-2340 \mathrm{~nm}$ for short wavelength infrared (SWIR). Good condition asphalt has the lowest value in the VIS2 ratio and highest value in the SWIR ratio. The converse is true for bad condition asphalt. Another approach for identifying different states of material is to use hydrocarbon seeps. These features are typically the maximum absorption bands situated around the wavelength of $1730 \mathrm{~nm}$ and $2300 \mathrm{~nm}$ (Clutis, 1989). The degree of oily components existing in asphalt is one of the factors creating different conditions since this characteristic influences the molecular structure and consequently the degree of viscosity of the asphalt surface. The higher the oily components, the more viscous the road surface and in turn the stronger the hydrocarbon absorption bands. Deeper absorption bands indicate better condition of the asphalt surface material (Figure 9). As the asphalt gets older (condition of the asphalt gets worse), the degree of viscosity reduces and it becomes prone to cracks (Weng Q., 2008). As a result, the reflectance of the surface increases and eventually the hydrocarbon bands become weak and approximate a straight line. This means that the condition of asphalt is bad. The results of the investigation indicate that the mean function is more reliable for identifying different conditions of asphalt. The classification result for the whole study area based on mean function is shown in figure 10. The classification statistics indicate the $23 \%$ of the pixels are identified as good asphalt, $23 \%$ as intermediate and $14 \%$ as bad asphalt. The remaining pixels are unclassified. The statistics are with respect to the area covered by roads only. Figure 11 shows an example of part of Steinbeis road which is classified as bad. Spectra of corresponding locations from the HyMap data supports the result obtained. Additionally, field investigation confirms that the condition of the asphalt for the road is bad. 


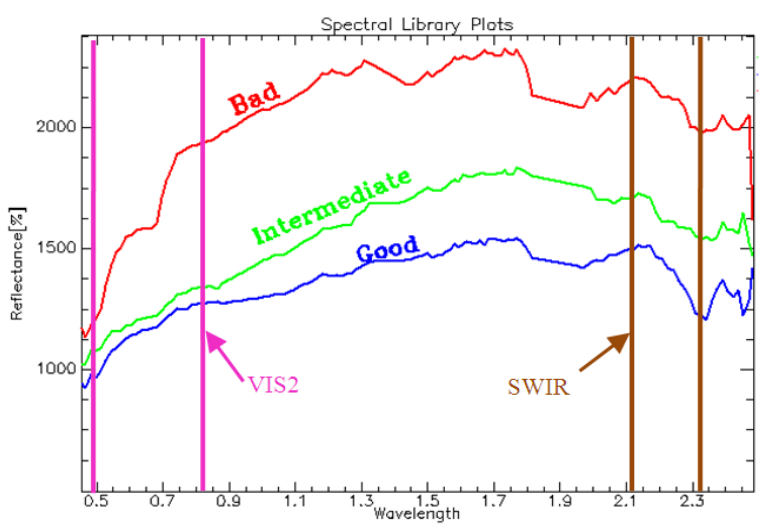

Figure 8. VIS2 and SWIR ratio ranges.

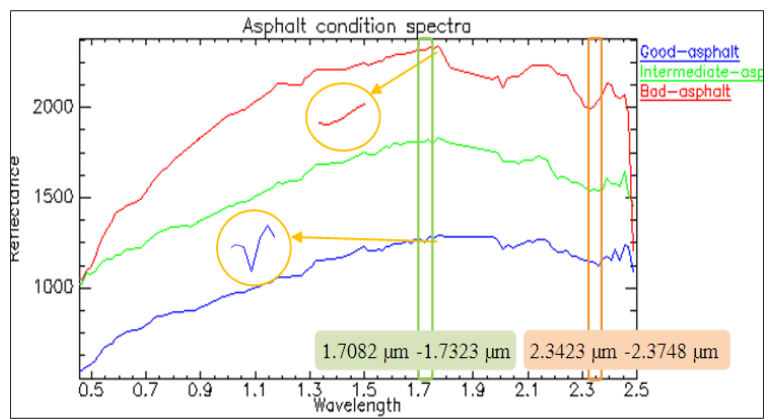

Figure 9. Hydrocarbon absorption band ranges.

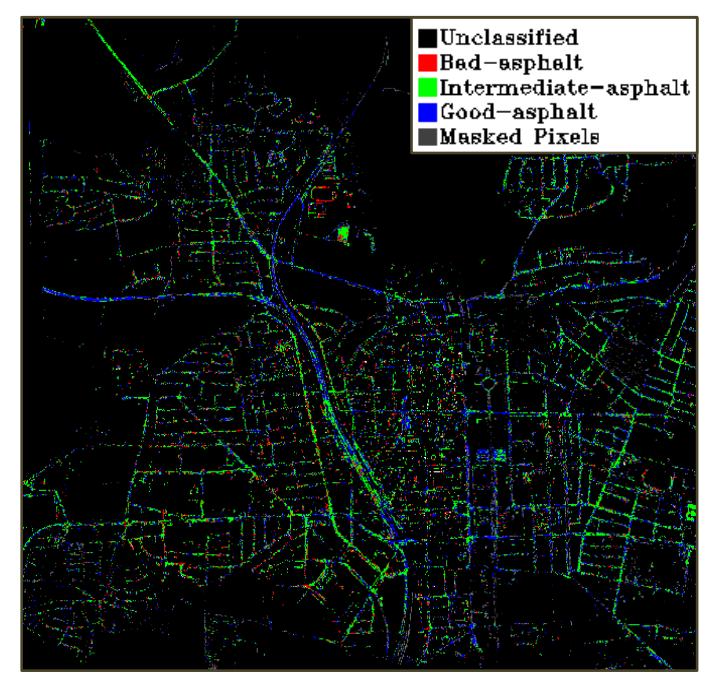

Figure 10. Classification result for the whole study area.
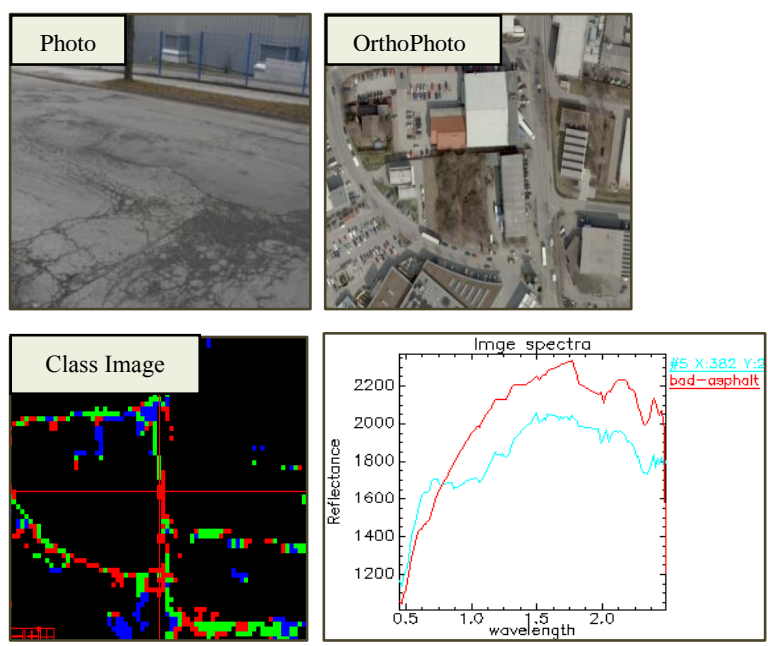

Figure 11. Assessment of classification result on Steinbeisstraße.

Even though a comprehensive evaluation is not yet finished, the spot checks supported by field visits indicate a high potential of the approach for identifying roads with good, intermediate and bad surface condition.

\section{CONCLUSION}

This study focuses on two main purposes, namely identification of road surface materials and investigation of different conditions of asphalt. The classification results show that the SAM classification based on regions of interest is helpful for discriminating road surface materials. Regions of interest represent the mean spectrum for an area of interest and thus take into account variations in spectra of materials due to age or usage. Additionally, combining mean and standard deviation spectral functions is helpful for distinguishing asphalt, concrete and gravel. This is possible since asphalt has a lower mean value compared to the other two materials and concrete has a lower standard deviation than gravel over the wavelength range of $619.9 \mathrm{~nm}$ $1323.7 \mathrm{~nm}$. In terms of condition determination for asphalt roads, it is observed that the mean function gives reliable results with good success in identifying roads with good, intermediate and bad surface condition. This is because the spectra of different conditions of asphalt differ significantly in albedo. From the research, it was observed that hydrocarbon absorption bands are useful in surface material condition investigation. In particular the wavelength range $1.7082 \mu \mathrm{m}$ to $1.7323 \mu \mathrm{m}$ are suitable for identifying different states of asphalt.

It is observed that the number of unclassified pixels in the results presented in this paper is generally significant. Therefore, more research should be done to improve the methods adopted and thus reduce the number of unclassified pixels. Additionally, hyperspectral data with better spatial resolution should be used. 


\section{References}

Bhaskaran, S. \& Datt, B., 2000. Applications of hyperspectral remote sensing in urban regions.

http://www.gisdevelopment.net/aars/acrs/2000/ps1/ps112pf.htm (24 Nov. 2011).

Clutis, E.A.,1989. Spectral reflectance properties of hydrocarbons, remote sensing implementation. Science, Vol. 245, no.4914, pp.165-168.

Goetz, A.F.H., Vane, G., Solomon, J.E. \& Rock, B.N., 1985. Imaging spectrometry for earth remote sensing: Science, Vol. 228 (4704), pp. 1147-1153.

Heiden, U., Segl, K., Rossner, S. \& Kaufmann, H., 2007. Determination of robust spectral features for identification of urban surface materials in hyperspectral remote sensing data, Remote Sensing of Environment, 111(4), pp. 537-552.

Heiden, U., Segl, K., Rossner, S. \& Kaufmann, H., 2005. Determination and verification of robust spectral features for an automated classification of sealed urban surfaces. In: Zagajewski, B. and Sobczak, M., (Eds.), Imaging Spectroscopy: New quality in environmental studies, pp.165-174.

Herold, M., \& Roberts, D., 2005: Spectral characteristics of asphalt road aging and deterioration: implications for remotesensing applications. Applied Optics, 44(20), pp.4327-4334.

Mohammadi, M., 2011. Road Classification and Condition Determination using Hyperspectral Imagery. Master thesis, University of Applied Sciences Stuttgart, Germany.

Noronha, V., Herold, M., Gardner, M. \& Roberts, D.A., 2002. Spectrometry and Hyperspectral Remote Sensing for Road Centerline Extraction and Evaluation of Pavement Condition. Proceedings of the Pecora Conference, Denver, CO.

Segl, K., Heiden, U., Mueller, M. \& Kaufmann, H., 2003. Endmember detection in urban environments using hyperspectral HyMap data. Third EARSeL Workshop on Imaging Spectroscopy,Herrsching.

Van der Meer, F., 2004. Analysis of spectral absorption features in hyperspectral imagery. International Journal of Applied Earth Observation and geoinformation, 5(1), pp. 55-68.

Weng, Q., 2008. Remote sensing of impervious surfaces. CRC Press/Taylor and Francis in press, United States of America, pp. 238-241.

Zhang, Q. \& Couloigner, I., 2004. A Framework for Road Change Detection and Map Updating. In: International Archives of the Photogrammetry, Remote Sensing and Spatial Information Sciences, Istanbul, Turkey, Vol. 35, Part B2, pp.720-734. 